\title{
Amplification de puissance en régime non linéaire et en hautes fréquences à transistor MOS
}

\author{
S. Latreche $(*)$, G. Tardivo (**), M. Belabadia (*) et P. Rossel (*)
}

(*) Laboratoire d'Automatique et d'Analyse des Systèmes du Centre National de la Recherche Scientifique, 7, avenue du Colonel-Roche, 31077 Toulouse Cedex, France

$\left.{ }^{* *}\right)$ Thomson Semiconducteurs, Centre de Tours, rue Pierre-et-Marie-Curie, 37001 Tours Cedex, France

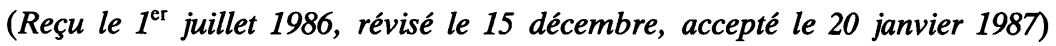

Résumé. - Un modèle mathématique du transistor V.MOS prenant en compte les effets de canal court et les non-linéarités du composant est proposé. Il est utilisable en régime i) continu, ii) dynamique sinusoïdal petit signal, iii) et il permet, de plus, la simulation du régime alternatif grand signal. Il sert de base à une méthodologie originale permettant la conception et la simulation par ordinateur d'amplificateurs R.F. de puissance (bande V.H.F.) à transistors MOS.

\begin{abstract}
A mathematical model of V.MOS transistor, taking into account the short channel effects and the non-linearities in the transistor is proposed. It enables the study of V.MOS under direct current and small signal conditions. More, it can be used for large signal simulations and it is the base of new procedure for C.A.D. of power radio-frequency amplifiers (V.H.F. band) using power MOS transistors.
\end{abstract}

\section{Introduction.}

Depuis son apparition sur le marché des composants discrets en 1976, le transistor MOS de puissance s'est imposé comme composant majeur, du fait de ses nombreux avantages par rapport aux composants bipolaires. En ce qui concerne les applications en haute fréquence, le transistor MOS présente notamment une grande stabilité thermique et un gain élevé [4-5].

Compte tenu de ces propriétés, il est devenu nécessaire de pouvoir simuler le fonctionnement des transistors MOS en régime haute fréquence, afin de pouvoir prévoir leurs caractéristiques et de concevoir les circuits d'amplification les utilisant.

Les méthodes proposées à ce jour reposent, soit sur la mesure des paramètres dynamiques $Y_{i j}$ ou $S_{i j}$, soit sur le calcul des éléments physiques, mais ne permettent la simulation du composant qu'en régime de petit signal [6-7]. La simulation en forte puissance est, en effet beaucoup plus délicate du fait du comportement très non linéaire du transistor et de l'importance des circuits extérieurs.

Les travaux présentés ici visent précisément à décrire une procédure originale, qui, à partir d'un modèle mathématique non linéaire du transistor MOS, permet l'étude des performances du compo- sant, tant en petit qu'en grand signal, ainsi que la conception assistée par ordinateur d'amplificateurs haute fréquence de puissance.

\section{Modèle mathématique du transistor V.MOS.}

La coupe du transistor V.MOS est présentée à la figure 1. Le modèle que nous proposons prend en compte d'une part la zone active du canal, et d'autre

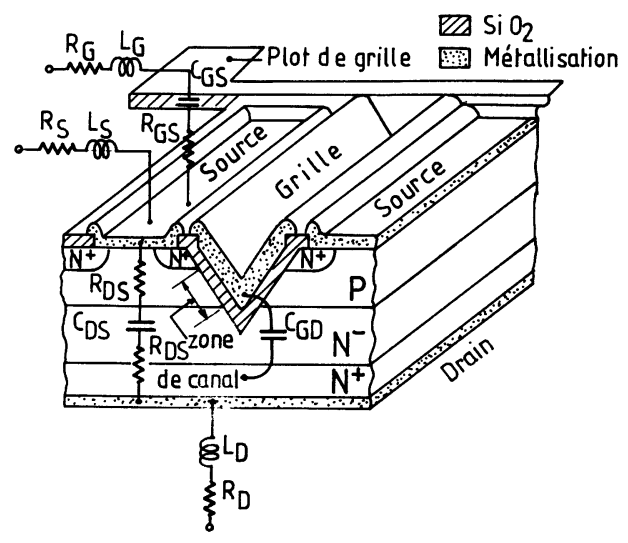

Fig. 1. - Coupe schématique du transistor V.MOS.

[Cross section of V.MOS transistor.] 
part, les éléments «parasites » liés à la structure même du transistor.

2.1 Modélisation DE LA ZONE ACTIVE [13-14]. Le modèle utilisé repose sur la résolution mathématique des équations régissant la zone active du transistor (équation de Poisson, neutralité électrique). Ces équations ont été souvent étudiées sous diverses formes [8-11]. Néanmoins, l'originalité de l'approche dynamique repose dans la prise en compte de l'effet dit de canal court, c'est-à-dire la réduction de la mobilité sous l'action des champs longitudinal et transversal. En effet, étant donné que le champ source-drain peut dans un transistor MOS atteindre des valeurs très élevées, de l'ordre de $10^{6} \mathrm{~V} / \mathrm{cm}$, le mouvement des porteurs du canal, sous l'action de ce champ, ne pourra pas être décrit par une loi simple caractérisée par une mobilité constante. Plusieurs analyses théoriques [1, 3, 24-27] et diverses relations empiriques ont été proposées pour rendre compte des variations locales de la mobilité des électrons en fonction des composantes du champ électrique. Pour décrire les influences des champs transversaux et longitudinaux, nous supposerons que chaque champ agit selon une loi hyperbolique sur la mobilité effective des porteurs dans le canal, ce qui conduit, pour les électrons, à la relation suivante [12], qui est à ce jour une des dépendances communément admise et validée dans le cas des transistors MOS dits à canal court [3] :

$$
\mu=\frac{\mu_{0}}{\left[1+\frac{V_{\mathrm{G}}^{\prime}-\Phi_{y}}{\psi}\right]\left[1+\frac{\mathrm{d} \Phi_{y}}{E_{0} \mathrm{~d} y}\right]}
$$

où $\mu_{0}$ est la mobilité à champ faible, $E_{0}$ le champ critique longitudinal, $\psi$ le potentiel qui traduit les effets du champ transversal, $\Phi_{y}$ le potentiel en surface, $V_{\mathrm{G}}^{\prime}$ la tension effective de grille définie par :

$$
V_{\mathrm{G}}^{\prime}=V_{\mathrm{G}}+Q_{\mathrm{ss}} / C_{\mathrm{ox}}-\Phi_{\mathrm{ms}}
$$

avec $Q_{\text {ss }}$ la charge totale des états de surface ramenée à l'interface $\mathrm{S}_{i} / \mathrm{S}_{i} \mathrm{O}_{2}, \Phi_{\mathrm{ms}}$ le travail de sortie métal-semiconducteur, $C_{\text {ox }}$ la capacité d'oxyde par unité de surface.

Le courant drain continu s'exprime alors en zone non saturée sous la forme [12]:

$$
\begin{aligned}
& I_{\mathrm{d}}=\frac{\mu_{0} \cdot Z / L \cdot C_{\mathrm{ox}} \cdot \psi}{1+V_{\mathrm{D}} /\left(L \cdot E_{0}\right)}\left[V_{\mathrm{D}}+\psi \operatorname{Ln}\left[\frac{\psi+V_{\mathrm{G}}^{\prime}-2 \cdot \Phi_{\mathrm{F}}-V_{\mathrm{D}}}{\psi+V_{\mathrm{G}}^{\prime}-2 \cdot \Phi_{\mathrm{F}}}\right]+2 \cdot\left(\Phi_{\mathrm{B}}\right)^{1 / 2} \times\right. \\
& \times\left[\left(V_{\mathrm{D}}+2 \Phi_{\mathrm{F}}\right)^{1 / 2}-\left(2 \cdot \Phi_{\mathrm{F}}\right)^{1 / 2}-\left(V_{\mathrm{G}}^{\prime}+\psi\right)^{1 / 2} \cdot\left\{\operatorname{Argtanh}\left[\left(V_{\mathrm{D}}+2 \Phi_{\mathrm{F}}\right) /\left(V_{\mathrm{G}}^{\prime}+\psi\right)\right]^{1 / 2}\right.\right. \\
& \left.\left.\left.-\operatorname{Argtanh}\left[\left(2 \Phi_{\mathrm{F}}\right) /\left(V_{\mathrm{G}}^{\prime}+\psi\right)\right]^{1 / 2}\right\}\right]\right] \text {. }
\end{aligned}
$$

Avec

$$
\Phi_{\mathrm{B}}=\frac{2 \cdot q \cdot N_{\mathrm{A}} \cdot \varepsilon_{0} \cdot \varepsilon_{S_{i}}}{.\left(C_{\mathrm{ox}}\right)^{2}}
$$

et

$$
\Phi_{\mathrm{F}}=\frac{k T}{q} \cdot \operatorname{Ln}\left[N_{\mathrm{A}} / n_{\mathrm{i}}\right] .
$$

Dans ces relations, $q$ est la charge électronique, $n_{\mathrm{i}}$ la concentration intrinsèque, $Z$ la largeur de grille, $L$ la longueur du canal, $C_{\text {ox }}$ la capacité d'oxyde par unité de surface, $k T / q$ le potentiel thermodynamique, $\varepsilon_{0} \cdot \varepsilon_{\mathrm{SI}}$ la constante diélectrique du silicium et $N_{\mathrm{A}}$ le dopage supposé uniforme, dans la zone de canal.

La tension de pincement $V_{\mathrm{P}}$ est obtenue par une dérivation du courant de drain $I_{\mathrm{d}}$ par rapport à la tension de drain $V_{\mathrm{D}}$, ce qui fournit la relation implicite suivante :

$$
\begin{aligned}
& \mu_{0} \cdot \frac{Z}{L} \cdot C_{\mathrm{ox}} \cdot \frac{1}{1+\frac{V_{\mathrm{G}}^{\prime}-V_{\mathrm{P}}-2 \cdot \Phi_{\mathrm{F}}}{\psi}} \times \\
& \times\left\{V_{\mathrm{G}}^{\prime}-V_{\mathrm{P}}-2 \cdot \Phi_{\mathrm{F}}-\left[\Phi_{\mathrm{B}} \cdot\left(V_{\mathrm{P}}-2 \cdot \Phi_{\mathrm{F}}\right)\right]^{1 / 2}\right\} \\
&-I_{\mathrm{d}} /\left(L \cdot E_{0}\right)=0 .
\end{aligned}
$$

Cette relation est résolue par méthode de dichotomie. Nous admettons, dans le cas du transistor MOS considéré, dans lequel le dopage de drain est plus faible - d'une décade environ - que la zone $\mathrm{P}$ de canal que les effets de modulation de longueur effective sont peu marqués [36]. L'hypothèse de saturation parfaite est donc considérée. Ainsi le courant de drain en régime saturé $I_{\mathrm{DSAT}}$ est obtenu en remplaçant dans la relation (2), la tension de drain par $V_{\mathrm{P}}$.

En régime dynamique sinusoïdal, les équations différentielles régissant le courant $i(y, t)$ et la variation de tension entre grille et canal $\varepsilon(y, t)$ sont fournies par le système suivant dont la méthode d'obtention a été décrite dans les références $[13,22]$ :

$$
\begin{gathered}
\frac{\mathrm{d}^{2} i(y, p)}{\mathrm{d} V_{0}(y)^{2}}-\frac{\mu_{0} \cdot\left[Z \cdot C_{\mathrm{ox}}\right]^{2} \cdot \psi \cdot p}{\left[I_{\mathrm{d}}\right]^{2}} \times \\
\quad \times \frac{V_{0}(y)}{V_{0}(y)+\psi} \cdot i(y, p)=0 \\
\varepsilon(y, p)=\frac{B_{0}\left[V_{0}(y)\right]}{p^{\prime}} \cdot \frac{\mathrm{d} i(y, p)}{\mathrm{d} V_{0}(y)}
\end{gathered}
$$


avec $p=j \cdot w, w$ la pulsation et

$$
p^{\prime}=\left(\frac{\mu_{0} \cdot\left[Z \cdot C_{\mathrm{ox}}\right]^{2} \cdot V_{0}^{3}(0)}{\left[I_{\mathrm{d}}\right]^{2}} \cdot p\right)
$$

et

$$
\begin{aligned}
& B_{0}\left[V_{0}(y)\right]=\frac{V_{0}^{3}(0)}{I_{\mathrm{d}} \cdot \psi} \times \\
& \times\left[\frac{V_{0}(y)}{\psi+V_{0}(y)}-\frac{I_{\mathrm{d}}}{\mu_{0} \cdot Z \cdot C_{\mathrm{ox}} \cdot \psi \cdot E_{0}}\right]^{-1}
\end{aligned}
$$

et $V_{0}(y)$ est la tension continue grille-canal au point d'abscisse $y$ du canal. $i(y, t)$ et $\varepsilon(y, t)$ représentent les variations dynamiques de ces quantités. Soit

$$
\begin{aligned}
& I(y, t)=I_{\mathrm{d}}+i(y, t) \\
& V(y, t)=V_{0}(y)+\varepsilon(y, t) .
\end{aligned}
$$

Les solutions des équations (3) et (4) sont obtenues soit par recherche de solutions approchées [23] utilisant la méthode WBKJ, soit par des développements en série [13]. Cette méthode plus rigoureuse est une généralisation des calculs effectués dans le cas d'une mobilité constante par A. Van Der Ziel et J. W. Ero [19] et des travaux effectués par E. N. Pronontarios [20]. Elle consiste à rechercher des solutions sous la forme :

$$
i(y, p)=\sum_{n=0}^{\infty} a_{n} \cdot V_{0}^{n}(y) .
$$

En calculant les dérivées première et seconde et en reportant dans l'équation (3), on détermine par identification les coefficients $a_{n}$ en fonction de $a_{0}$ et $a_{1}$. Le détail des développements mathématiques pour obtenir ces solutions est décrit dans les références [22] et [23].

En limitant ces développements au second ordre par rapport à $p^{\prime}$, on peut modéliser par synthèse de circuit, la zone active du transistor MOS sous forme d'un quadripôle actif constitué par un générateur de courant et des éléments discrets - résistifs et capacitifs - dont les valeurs ne dépendent que des paramètres géométriques et physiques et des tensions instantanées sur les électrodes. Le détail de ce développement mathématique, puis la synthèse classique par quadripôle des réseaux de deuxième ordre sont détaillés dans les références [13-15].

2.2 MODÈLE COMPLET DU TRANSISTOR V.MOS. Le schéma complet est obtenu en ajoutant à la zone active les éléments parasites dus au montage, au boîtier ou à la structure même du transistor. La localisation de ces différents éléments sur une coupe du transistor est représentée à la figure 1 dans le cas du V.MOS. Ces éléments parasites sont les résistances d'accès $R_{\mathrm{G}}, R_{\mathrm{S}}, R_{\mathrm{D}}$, la résistance $R_{\mathrm{DS}}$ du substrat $\mathrm{P}$ et du drain $\mathrm{N}^{-}$sous la capacité $C_{\mathrm{DS}}$ et la résistance du substrat $R_{\mathrm{GS}}$ sous la métallisation de grille sous l'oxyde épais, les capacités de transition $C_{\mathrm{DS}}$, de recouvrement $C_{\mathrm{GD}}$ et de plot de contact $C_{\mathrm{GS}}$, ainsi que les inductances $L_{\mathrm{G}}, L_{\mathrm{S}}, L_{\mathrm{D}}$, liées aux interconnexions. Ces éléments peuvent être évalués en tenant compte de la géométrie, de la technologie de fabrication et de mesures spécifiques dont les principes et mises en œuvre ont été explicités, de façon générale, antérieurement [31] et mis en œuvre dans le cas particulier du transistor MOS de puissance par L. Belabas [13], S. Latreche [14], J. G. Mena [6], P. McGregor [7] et M. du Plessis [28]. Leurs valeurs pour un transistor de type VMP4 sont spécifiées par exemple dans le tableau I [13]. Le schéma complet obtenu de ce produit est présenté dans la figure 3.

Dans le modèle que nous utilisons, ces éléments parasites ont une valeur constante, à l'exception de la capacité drain-source $C_{\mathrm{DS}}$, qui varie en fonction des tensions comme une capacité de jonction abrupte selon la dépendance :

$$
C_{\mathrm{DS}}=\frac{C_{\mathrm{DS} 0}}{\left[1+V_{\mathrm{D}} / V_{\mathrm{bi}}\right]^{1 / 2}}
$$

où $V_{\mathrm{bi}}$ est la tension de diffusion de la diode drain. $C_{\text {DS0 }}$ est proportionnelle à l'aire de drain et à la racine carrée du dopage de la région $\mathrm{N}^{-}$.

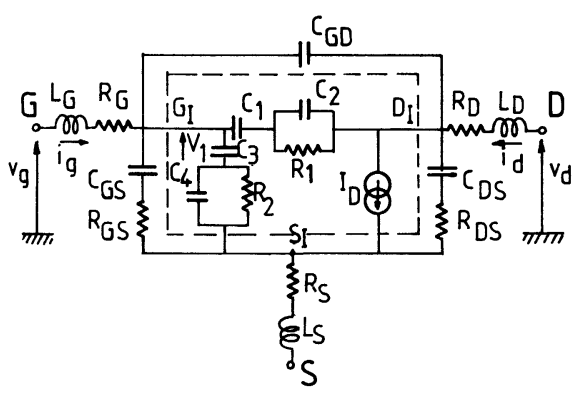

Fig. 2. - Modèle complet du transistor V.MOS $\left(C_{1}\right.$, $C_{2}, C_{3}, C_{4}, C_{\mathrm{DS}}, R_{1}, R_{2}$ et $I_{\mathrm{d}}$ dépendent des tensions).

[V.MOST equivalent circuit where $C_{1}, C_{2}, C_{3}, C_{4}$, $C_{\mathrm{DS}}, R_{1}, R_{2}$ and $I_{\mathrm{d}}$ are voltage dependent.]

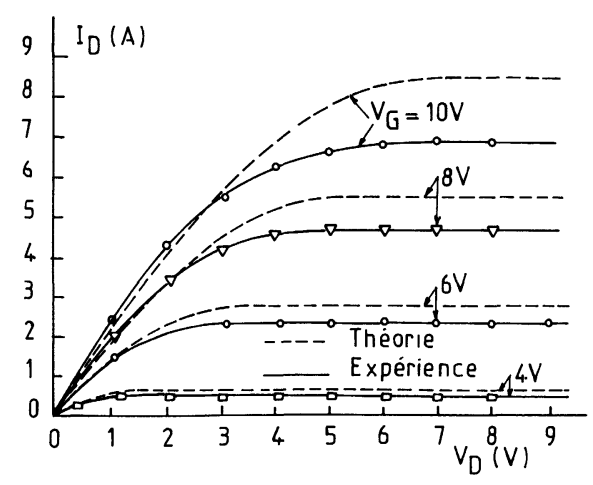

Fig. 3. - Caractéristiques $I_{\mathrm{d}}\left(V_{\mathrm{D}}\right)$. Transistor DV 1008. [D.C. Characteristics.] 
Tableau I. - Paramètres spécifiques au transistor VMP 4.

\begin{tabular}{|c|c|c|c|c|c|}
\hline $\begin{array}{c}Z \\
(\mathrm{~cm})\end{array}$ & $\begin{array}{c}L \\
(\mu \mathrm{m})\end{array}$ & $\begin{array}{c}\mu_{0} \\
\mathrm{~cm}^{2} / \mathrm{v} \cdot \mathrm{s}\end{array}$ & $\begin{array}{c}L E_{0} \\
(\mathrm{~V})\end{array}$ & $\begin{array}{c}C_{0 \times} \\
\left(\mathrm{nF} / \mathrm{cm}^{2}\right)\end{array}$ & $\begin{array}{c}V_{\mathrm{FB}} \\
(\mathrm{V})\end{array}$ \\
\hline 2,0 & 2,0 & 390 & 5,0 & 40 & $-1,2$ \\
\hline$R_{\mathrm{SG}}(\Omega)$ & $R_{\mathrm{DS}}(\Omega)$ & $R_{\mathrm{G}}(\Omega)$ & $R_{\mathrm{S}}(\Omega)$ & $R_{\mathrm{D}}(\Omega)$ & $\psi(\mathrm{V})$ \\
\hline 0,7 & 0,7 & 0,2 & 0,11 & 1,15 & 25,8 \\
\hline$L_{\mathrm{G}}(\mathrm{nH})$ & $L_{\mathrm{S}}(\mathrm{nH})$ & $L_{\mathrm{D}}(\mathrm{nH})$ & $C_{\mathrm{DS} 0}(\mathrm{pF})$ & $C_{\mathrm{GD}}(\mathrm{pF})$ & $C_{\mathrm{GS}}(\mathrm{pF})$ \\
\hline 0,32 & 0,91 & 0,11 & 300 & 3,5 & 32 \\
\hline
\end{tabular}

La résistance de drain est liée à la couche épitaxiée $\mathrm{N}^{-}-\mathrm{N}^{+}$. Elle prend en compte les effets de défocalisation des lignes de courant dans ce drain [31, 33, 34].

D'autre part, si les extensions des zones de charge d'espace sont faibles devant les dimensions du semiconducteur dans lequel elles se développent, nous pouvons considérer en première approximation que les résistances $R_{\mathrm{DS}}$ et $R_{\mathrm{GS}}$ sont indépendantes des conditions de polarisation.

2.3 VALIDATION DU MODÈLE EN RÉGIMES STATIQUE ET ALTERNATIF PETIT SIGNAL. - Le schéma dynamique ainsi obtenu a été implanté sur le logiciel de simulation des circuits linéaires et non linéaires Astec III [14-15]. Les simulations en régime continu et dynamique petit signal permettent de calculer les caractéristiques courant-tension $I_{\mathrm{d}}\left(V_{\mathrm{D}}\right)$, les paramètres admittance $Y_{i j}$ et les paramètres d'ondes $S_{i j}$ du transistor, son gain maximum en puissance (MAG), et le coefficient de stabilité de Linvill (Kl). Ces paramètres sont comparés aux valeurs expérimentales. La figure 3 présente à titre d'exemple les caractéristiques $I_{\mathrm{d}}\left(V_{\mathrm{D}}\right)$ et la figure 4 le paramètre

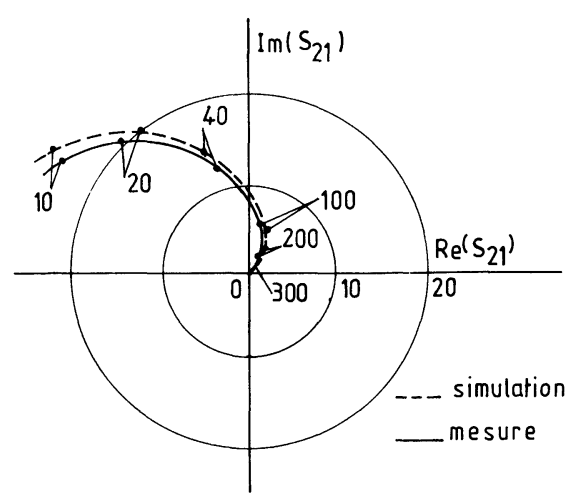

Fig. 4. - Paramètre $S_{21}$. Fréquence en $\mathrm{MHz}$. Transistor VMP4 $-V_{\mathrm{D}}=28 \mathrm{~V}, I_{\mathrm{d}}=0,8 \mathrm{~A}$.

$\left[S_{21}\right.$ parameter. Frequencies in $\mathrm{MHz}$.]
$S_{21}$. Sur les résultats obtenus, nous avons constaté que l'accord entre les simulations et les mesures est de l'ordre de $20 \%$, ce qui constitue une bonne validation du modèle. Notons que l'écart entre les caractéristiques $I_{\mathrm{d}}\left(V_{\mathrm{d}}\right)$ simulées et mesurées est dû en partie à l'effet de l'élévation de la température de cristal sur la vitesse des porteurs. Cet effet, particulièrement important à fort niveau de puissance [37, 38] n'est pas pris en compte dans ce modèle. Par contre si les effets de réduction de mobilité n'avaient pas été pris en compte, les écarts auraient pu être supérieurs à $100 \%$ aussi bien en régime statique [35] qu'en régime dynamique. Pour ce dernier régime, la référence [22] qui représente des variations comparatives théoriques des paramètres admittance lorsqu'on prend ou ne prend pas en compte ces effets de réduction corrobore ces écarts entre modèles.

\section{C.A.O. et simulation d'amplificateur radiofré- quence de puissance à transistor V.MOS.}

3.1 RAPPEL DES DÉFINITIONS. - L'application la plus importante et innovante de cette modélisation est la conception et la simulation d'amplificateurs de puissance en hautes fréquences. Le schéma général d'un amplificateur radiofréquence à bande étroite est donné à la figure 5 .

Il est composé d'un générateur de f.é.m. $E$, de fréquence $F$, et d'impédance interne $Z_{\mathrm{s}}$, d'un élé-

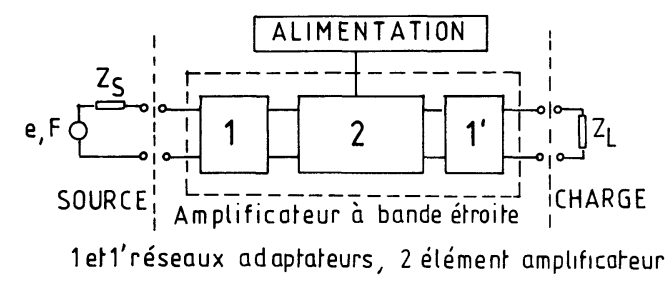

Fig. 5. - Configuration d'un amplificateur à bande étroite.

[Structure of a narrow band amplifier.] 
ment actif (le transistor V.MOS), d'une charge d'impédance $Z_{1}$ et de deux réseaux adapteurs.

Un tel amplificateur est optimisé si le transfert de puissance est maximal, d'une part entre la source et le transistor et d'autre part entre le transistor et la charge. Cette optimisation est assurée par les réseaux d'adaptation qu'il faut donc savoir calculer pour concevoir un amplificateur.

Les puissances que l'on peut définir en toute généralité dans un amplificateur de puissance sont i) la puissance retirable du générateur $P_{\text {ret }}$ définie par :

$$
P_{\text {ret }}=\frac{E^{2}}{4 \cdot \operatorname{Re}\left[Z_{\mathrm{s}}\right]}
$$

où $\mathrm{Re}$ désigne la partie réelle d'une impédance et ii) les puissances, $P_{\text {in }}$, à l'entrée et à la sortiẻ, $P_{\text {out }}$, du transistor calculées par les intégrales temporelles suivantes :

$$
\begin{aligned}
& P_{\text {in }}=\frac{1}{T} \int_{t}^{t+T} I g \cdot V g \mathrm{~d} t \\
& P_{\text {out }}=\frac{1}{T} \int_{t}^{t+T} I d \cdot V d \mathrm{~d} t
\end{aligned}
$$

où $I g, V g, I d, V d$ sont les courants et les tensions dynamiques respectivement sur la grille et sur le drain du transistor et $T$ la période des signaux.

Ces puissances sont calculées par intégration numérique en déterminant les valeurs temporelles $\operatorname{Ig}(t), V g(t), \operatorname{Id}(t), V d(t)$ au moyen du logiciel ASTEC III.

3.2 C.A.O. DES AMPLIFICATEURS. - Comme nous venons de le voir, le transistor est adapté en puissance si le maximum de puissance lui est fourni en entrée, et s'il débite le maximum de puissance dans la charge. Il est bien connu que ceci n'est possible que si le transistor «voit » à ses bornes les impédances conjuguées de ses impédances internes ; c'est-à-dire une impédance $Z_{\mathrm{s}}=Z_{\text {in }}^{*}$ en entrée et une impédance $Z_{1}=Z_{\text {out }}^{*}$ en sortie $\left(Z_{\text {in }}\right.$ et $Z_{\text {out }}$ étant les impédances d'entrée et de sortie du transistor). Ce sont ces impédances $Z_{\mathrm{s}}$ et $Z_{1}$ qui déterminent les configurations des réseaux d'adaptation et ce sont donc elles qu'il convient de calculer pour concevoir ensuite ces réseaux.

Deux modes de fonctionnement distincts sont alors possibles. Si le transistor fonctionne dans un régime linéaire (c'est-à-dire où le principe de superposition est applicable), nous dirons qu'il est dans un régime petit signal. Au contraire, si le principe de superposition n'est plus applicable, c'est-à-dire si le fonctionnement du transistor est très non linéaire, nous parlerons de régime grand signal. La notion de grand signal s'applique à la fois aux amplificateurs de classe $A$ en régime de compression et aux amplificateurs de classes $B$ et $C$ quel que soit le niveau des signaux.
3.2.1 Régime petit signal. - En petit signal, les impédances $Z_{\mathrm{s}}$ et $Z_{1}$ sont aisément calculables à partir des paramètres $Y_{i j}$ ou $S_{i j}$ du transistor. On a très classiquement :

$$
\begin{aligned}
& \operatorname{Re}\left(Y_{\mathrm{s}}\right)= \frac{1}{2 \cdot \operatorname{Re}\left(Y_{22}\right)}\left\{\left[2 \cdot \operatorname{Re}\left(Y_{11}\right) \cdot \operatorname{Re}\left(Y_{22}\right)-\right.\right. \\
&\left.\left.-\operatorname{Re}\left(Y_{12} \cdot Y_{21}\right)\right]^{2}-\left|Y_{12} \cdot Y_{21}\right|^{2}\right\}^{1 / 2} \\
& \operatorname{Im}\left(Y_{\mathrm{s}}\right)=-\operatorname{Im}\left(Y_{11}\right)+\frac{\operatorname{Im}\left(Y_{12} \cdot Y_{21}\right)}{2 \cdot \operatorname{Re}\left(Y_{22}\right)} \\
& \operatorname{Re}\left(Y_{1}\right)= \frac{1}{2 \cdot \operatorname{Re}\left(Y_{11}\right)}\left\{\left[2 \cdot \operatorname{Re}\left(Y_{11}\right) \cdot \operatorname{Re}\left(Y_{22}\right)-\right.\right. \\
&\left.\left.-\operatorname{Re}\left(Y_{12} \cdot Y_{21}\right)\right]^{2}-\left|Y_{12} \cdot Y_{21}\right|^{2}\right\}^{1 / 2} \\
& \operatorname{Im}\left(Y_{1}\right)=-\operatorname{Im}\left(Y_{22}\right)+\frac{\operatorname{Im}\left(Y_{12} \cdot Y_{21}\right)}{2 \cdot \operatorname{Re}\left(Y_{11}\right)}
\end{aligned}
$$

$\operatorname{Re}\left(Y_{i j}\right)$ et $\operatorname{Im}\left(Y_{i j}\right)$ représentent respectivement les parties réelles et imaginaires des paramètres admittance $Y_{i j} \cdot Y_{\mathrm{s}}$ et $Y_{1}$ sont les inverses de $Z_{\mathrm{s}}$ et $Z_{1}$.

3.2.2 Régime grand signal. - En grand signal, ce calcul n'est plus valable car on ne peut pas définir les paramètres $Y_{i j}$ dans ce régime. Les impédances «d'adaptation » doivent alors être déterminées comme étant celles qui permettent le transfert de puissance maximal d'une part de la source vers le transistor, puis d'autre part du transistor vers la charge. Ces impédances sont donc celles qui permettent d'obtenir la puissance de sortie $P_{\text {out }}$ maximale pour une puissance retirable fixée. La méthode utilisée pour déterminer numériquement ces impédances est la suivante :

i) En entrée, la valeur de l'impédance $Z_{\mathrm{s}}$ est gardée tout d'abord constante et égale à sa valeur petit signal.

ii) La partie réelle de l'impédance de sortie grand signal $\operatorname{Re}\left(Z_{1}\right)$ est obtenue en traçant les variations de $P_{\text {out }}$ en fonction de cette partie réelle pour différentes puissances retirables $P_{\text {ret }}$ à l'entrée. La partie imaginaire de $Z_{1}$ est alors gardée constante et égale à sa valeur petit signal. Cette famille de courbes est présentée à la figure 6. Il apparaît que ces courbes présentent toutes un maximum pour une valeur particulière de $\operatorname{Re}\left(Z_{1}\right)$, et que cette valeur diminue lorsque la puissance $P_{\text {ret }}$ augmente.

iii) La partie imaginaire de l'impédance de sortie Im $\left(Z_{1}\right)$ est déterminée de la même façon en maintenant $\operatorname{Re}\left(Z_{1}\right)$ la valeur précédemment obtenue en ii). Les courbes sont représentées sur la figure 7. On note que ces courbes présentent elles aussi un maximum en fonction de $\operatorname{Im}\left(Z_{1}\right)$ et que les valeurs de $\operatorname{Im}\left(Z_{1}\right)$ donnant ce maximum sont sensiblement constantes et égales à la valeur prise en petit signal.

iv) On peut ensuite itérer en faisant varier la valeur de l'impédance $Z_{\mathrm{s}}$ pour affiner l'optimisation. 


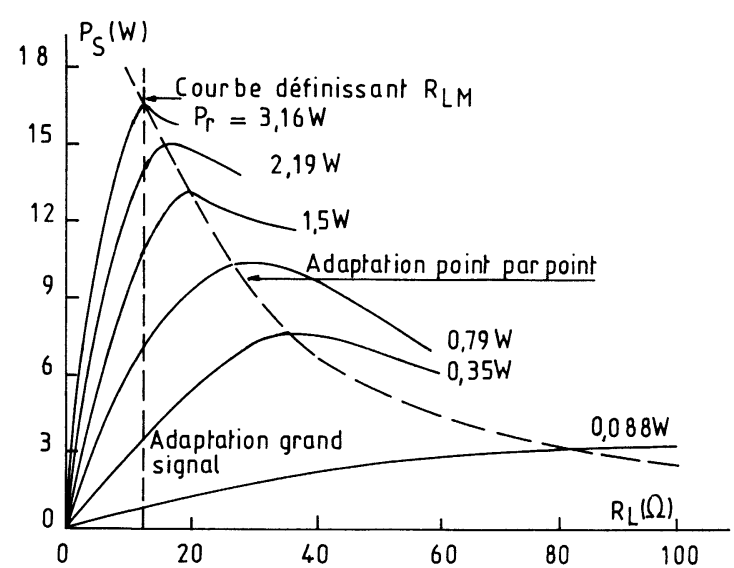

Fig. 6. - Evolution de la puissance de sortie en fonction de la résistance de charge $R_{\mathrm{L}}$, paramétrée par la puissance retirable $\left(V_{\mathrm{D}}=28 \mathrm{~V}, I_{\mathrm{d}}=0,65 \mathrm{~A}, F=175 \mathrm{MHz}\right.$. Transistor VMP4).

[Output power versus load resistance $R_{\mathrm{L}}$.]

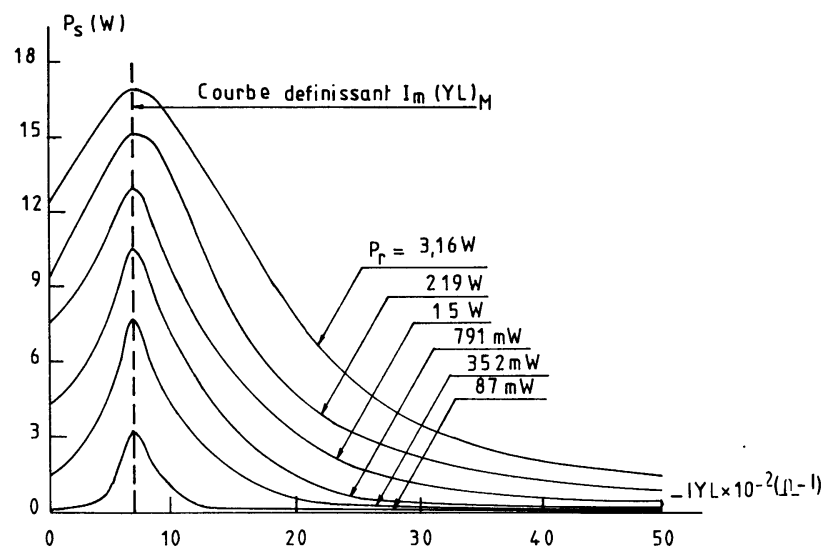

Fig. 7. - Evolution de la puissance de sortie en fonction de l'admittance de charge $Y_{\mathrm{L}}$, paramétrée par la puissance retirable $\left(V_{\mathrm{D}}=28 \mathrm{~V}, I_{\mathrm{d}}=0,65 \mathrm{~A}, F=175 \mathrm{MHz}\right.$. Transistor VMP4).

[Output power versus load inductance $Y_{\mathrm{L}}$ ]

De fait, on constate également que l'optimum de $Z_{\text {s }}$ coïncide pratiquement avec la' valeur «petit signal », c'est-à-dire que cette impédance varie peu, de $10 \%$ environ, en fonction du niveau de puissance. La référence [14] explicite les valeurs de la désadaptation en entrée en fonction du niveau de puissance d'entrée obtenues en conservant constante cette impédance.

Cette méthode permet donc en toute généralité de déterminer les impédances d'adaptation en "grand signal ». A partir de ces impédances, il est aisé de calculer par synthèse de circuits les configurations des réseaux non dissipatifs d'adaptation pour le transistor considéré [17].
3.3 ETUDE ET SIMULATION DES AMPLIFICATEURS. - En application directe de ces logiciels, divers amplificateurs ont été conçus et simulés par ordinateur, puis réalisés et testés expérimentalement dans les bandes des 30 à $200 \mathrm{MHz}$ et pour des puissances de sortie de 15 à $120 \mathrm{~W}$ environ sur des composants alimentés sous 13 ou $28 \mathrm{~V}$ [14, 16], ce qui correspond à ce jour aux gammes de fréquences et de puissances usuellement disponibles sur des transistors au niveau industriel. Des composants «multi-puces » de puissances plưs élevées pourraient éventuellement être envisagés. Ces valeurs en fréquence, $200 \mathrm{MHz}$, et en puissance, $120 \mathrm{~W}$, sont liées à ce jour, d'une part aux limitations créées par la technologie utilisée rendement, taille des puces, coût des produits - et d'autre part au choix de la configuration géométrique du transistor MOS de puissance - effets thermiques, contraintes de montage dans des boîtiers spéciaux à oxyde de béryllium et optimisés en fréquence.

Rappelons l'allure typique des caractéristiques de transfert $P_{\mathrm{s}}\left(P_{\mathrm{e}}\right)$ dans le cas de la classe $\mathrm{A}$, où l'on distingue le fonctionnement dit linéaire (où $P_{\mathrm{s}}$ est directement proportionnelle à $P_{\mathrm{e}}$ ) et la «compression" où la puissance de sortie n'augmente plus selon la loi linéaire (Fig. 8). La compression traduit

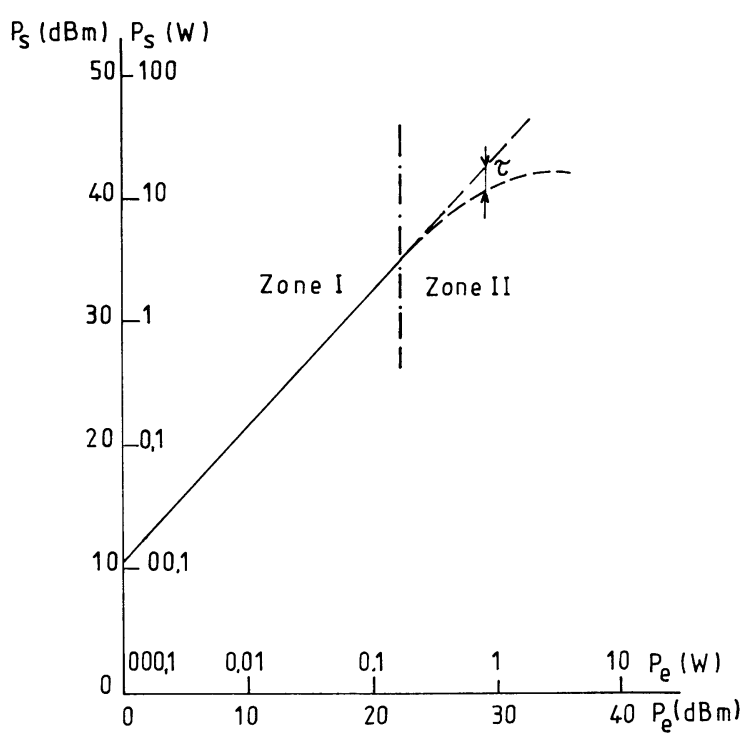

Fig. 8. - Allure de la caractéristique $P_{\mathrm{S}}\left(P_{\mathrm{E}}\right)$ en classe A.

[Typical $P_{\text {out }}\left(P_{\text {in }}\right)$ characteristics for class A.]

le comportement non linéaire du dispositif. Le modèle prend en compte les non-linéarités du transistor et rend compte de cet effet : les figures 9 et 10 comparent les mesures et les simulations à $150 \mathrm{MHz}$ sous $13 \mathrm{~V}$ d'alimentation pour les adaptations en petit et grand signal (c'est-à-dire que les réseaux d'adaptation sont calculés soit pour une faible puissance de sortie soit pour une puissance plus forte en zone de compression). Entre les deux régimes, il 


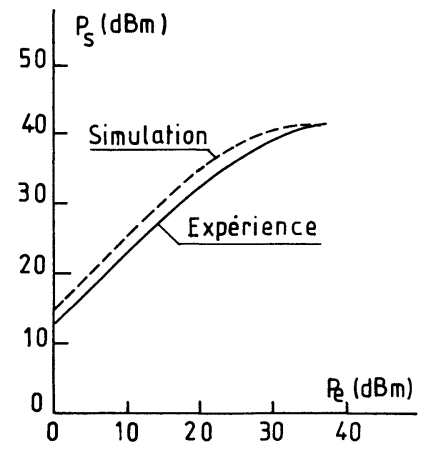

Fig. 9. - Caractéristiques $P_{\mathrm{S}}\left(P_{\mathrm{E}}\right)$. Adaptation petit signal $\left(V_{\mathrm{D}}=13 \mathrm{~V}, I_{\mathrm{d}}=0,6 \mathrm{~A}, F=175 \mathrm{MHz}\right.$. Transistor D 1240).

[ $P_{\text {out }}\left(P_{\text {in }}\right)$ characteristics. Small signal matching.]

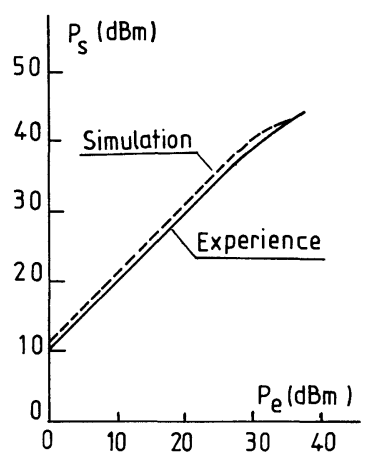

Fig. 10. - Caractéristiques $P_{\mathrm{S}}\left(P_{\mathrm{E}}\right)$. Adaptation grand signal $\left(V_{\mathrm{D}}=13 \mathrm{~V}, I_{\mathrm{d}}=0,6 \mathrm{~A}, F=175 \mathrm{MHz}\right.$. Transistor D 1240).

$\left[P_{\text {out }}\left(P_{\text {in }}\right)\right.$ characteristics. Large signal matching.]

apparaît une chute de gain, en zone linéaire, de près de $4 \mathrm{~dB}$, alors que le point de compression est au contraire accru de $8 \mathrm{~dB}$ environ lorsque une adaptation grand signal est utilisée.

Les figures 11 et 12 présentent cette même comparaison pour les différentes classes de polarisation : classe A $\left(I_{\mathrm{d}}=0,65 \mathrm{~A}\right)$, classe B $\left(I_{\mathrm{d}}=0,13 \mathrm{~A}\right)$, et classe $\mathrm{C}\left(I_{\mathrm{d}}=0 \mathrm{~A}\right)$ à $175 \mathrm{MHz}$ et sous $28 \mathrm{~V}$. On peut remarquer tout d'abord que les puissances de sortie sont du même ordre de grandeur, quelle que soit la polarisation, lorsque le composant est en régime de forte compression. Par contre, la valeur du gain petit signal, ainsi que la «linéarité » du composant (qui est l'écart de la pente par rapport à l'unité en coordonnées logarithmiques - puissances en $\mathrm{dBm}$ - de la caractéristique de transfert en puissance) se détériorent sensiblement lorsque l'on passe de la classe $\mathrm{A}$ à la classe $\mathrm{B}$, puis à la classe $\mathrm{C}$. De manière générale, il est apparu que l'accord entre les valeurs mesurées et celles simulées reste toujours inférieur à $3 \mathrm{~dB}$ dans tous les cas d'étude que nous avons traités.

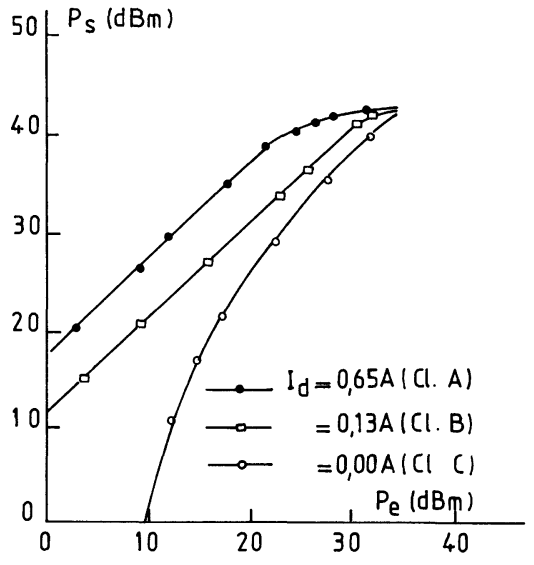

Fig. 11. - Caractéristiques $P_{\mathrm{S}}\left(P_{\mathrm{E}}\right)$ expérimentales $\left(V_{\mathrm{D}}=28 \mathrm{~V}, F=175 \mathrm{MHz}\right.$, transistor VMP4).

$\left[P_{\text {out }}\left(P_{\text {in }}\right)\right.$ experimental characteristics.]

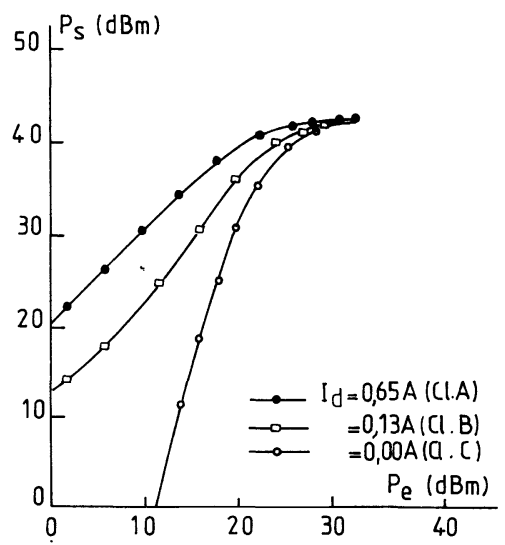

Fig. 12. - Caractéristiques $P_{\mathrm{S}}\left(P_{\mathrm{E}}\right)$ simulées $\left(V_{\mathrm{D}}=28 \mathrm{~V}\right.$, $F=175 \mathrm{MHz}$, transistor VMP4).

$\left[P_{\text {out }}\left(P_{\text {in }}\right)\right.$ theoretical characteristics.]

\section{Conclusion.}

Le but principal de cet article a été de décrire une méthodologie de simulation d'amplificateurs haute fréquence de puissance à transistor MOS. Sur la base de cette méthode, un ensemble de logiciels a été développé en vue de permettre l'analyse, tant en petit, qu'en grand signal des circuits amplificateurs à transistor MOS. Les résultats que nous avons présentés permettent de conclure que ces logiciels sont adaptés, tant pour la conception, que pour la simulation des amplificateurs. Ils évitent la réalisation souvent longue et coûteuse de maquettes en fournissant a priori les éléments caractéristiques de l'amplificateur. Enfin, ils sont un élément de conception du transistor lui-même dont il est possible de connaître, avant toute réalisation technologique, les caractéristiques en petit et surtout en grand signal. Ces résultats sont en cours d'extension d'une part aux composants D.MOS (optimisation de ces compo- 
sants et C.A.O. des amplificateurs les utilisant) et d'autre part au cas des quadripôles instables au sens de Linvill, c'est-à-dire aux circuits où le coefficient $\mathrm{K}$ de Linvill est inférieur à l'unité $[18,35]$.

\section{Bibliographie}

[1] Leburton, J. P., Borda, E. E., $v-E$ Dependence in small-sized MOS Transistors, IEEE Trans ED ED-29, N N 8 (1982) 1168-1171.

[2] RisCH, L., «Electron Mobility in Short-Channel MOSFET's », IEEE Trans ED, ED-30, $\mathrm{N}^{\circ} 8$ (1983) 959-961.

[3] Coen, R. W., Muller, R. S., « A Uniform Channel IGFET », Electronics Research Laboratory, College of Engineering, University of California, Berkeley, June 1979.

[4] Grant, D., Tregidga, «A., Power Mosfet's : Power for the $80 »$, Solid State Technol., 28, $\mathrm{N}^{\circ} 11$ (1985) 111-116.

[5] HARRIS, J. R. et al., « Design state of art accessed at R.F. frequencies », MSN C.T., 1985.

[6] Mena, J. G., High frequency performance of VD.MOS power transistors, M.A. Sc. Thesis, Toronto, 1981.

[7] MCGregor, P., Double diffused MOSFET's for CATV amplifiers, M.A. Sc. Thesis, Toronto, 1982.

[8] BuRns, J. R., « High Frequency Characteristics of Insulated Gate Field-Effect Transistors », $R C A$ Rev. 28 (1967) 385-418.

[9] Van Nielen, J. A., A simple and accurate approximation to the high frequency characteristics of insulated gate field-effect transistors, Solid State Electron 12 (1969) 826-829.

[10] DAS, M. B., « High frequency network properties of MOS transistor including the substrate resistivity effects », IEEE Trans. E.D., ED-16, $\mathrm{N}^{\circ} 12$ (1969) $1049-1069$.

[11] Haslett, J. W., Trofimenkoff, E. N., « Small signal high frequency equivalent circuit for the Metal-Oxyde-Semiconductor Field-Effect Transistors ", Proc. IEEE 116, N ${ }^{\circ} 5$ (1969) 699-702.

[12] VASSILIEFF, G., Modèle du transistor MOS. Influence des variations de la mobilité des porteurs, Thèse, Université Paul-Sabatier, Toulouse, 1971.

[13] Belabas, L., Propriétés en haute fréquence du transistor MOS à canal vertical, Thèse, Université Paul-Sabatier, Toulouse, 1983.

[14] LATRECHE, S., Le transistor MOS à canal vertical en régime non linéaire d'amplification haute fréquence de puissance, Thèse, Université PaulSabatier, Toulouse, 1985.

[15] Latreche, S., Tardivo, G., Belabadia, M., RosSEL, P., Modèle mathématique du transistor $V . M O S$ en régimes de commutation et d'amplification haute fréquence de puissance, Rapport LAAS N ${ }^{\circ}$ 85112, mai 1985.

[16] TARDIVO, G., Conception d'un amplificateur haute fréquence de puissance à transistor MOS, Rapport LAAS $\mathrm{N}^{\circ} 85353$, décembre 1985.

[17] «Solid State Power Circuits », RCA Designer's Handbook, Technical Series SP52, 1971, 430528.
[18] Rollet, J. M., « Stability and Power-Gain Invariants of linear Two ports", I.R.E. Trans. Circuit Theory (1962) 29-31.

[19] VAN DER ZIEL, A., ERO, J. W., «Small signal high frequency Theory of field effect transistor » IEEE Trans. ED (1964) 128-135.

[20] Pronontarios, E. N., Wing, O., « Computation of the step response of a general non uniform R.C. distribution network ", IEEE Trans C.T. 144, $\mathrm{N}^{\circ} 2$ (1967) 212-221.

[21] Gamboa, M., Rossel, P., Tranduc, H. et Phan PHAM, T., Le transistor MOS de puissance en régime de saturation : la résistance de saturation et les effets de faible multiplication, Revue Phys. Appl. 17 (1982) 65-74.

[22] Rossel, P., Guegan, G., Martinot, H., « Propriétés en haute fréquence des transistors MOS à canal court. Analyse théorique des propriétés de la zone active", Revue Phys. Appl. 14 (1979) 763-773.

[23] Rossel, P., « Méthode de Calcul des Paramètres Haute Fréquence des Transistors MOS en Régime de non-pincement », Electron. Lett. 8, $\mathrm{N}^{\circ} 25$ (1972) 614-616.

[24] Chu Hao, Cabon Tili, B., Cristoloveanu, S., GHIBAUdo, G., «Experimental determination of short channel MOSFET parameters », Solid State Electron 28, $\mathrm{N}^{\circ} 10$ (1985) 1025-1030.

[25] Ghibaudo, G., An analytical Model of Conductance and Transconductance for Enhanced Mode MOSFET's, Phys. Status Solidi (a) 95 (1986) 323.

[26] Sun, S. C., Plummer, J. D., « Electron mobility in inversion and accumulation layers on thermally oxidized silicon surfaces", IEEE Trans. ED, ED 27 (1980) 671-683.

[27] FunG, F. F., Fowler, A. B., « Hot electron effects and saturation velocities in silicon inversion layers », J.A.P. 41 (1970) 1825-1831.

[28] Du Plessis, M., « Die ontwerp en realisering van MOS U.H.F. drywingstransistors », Proefskrif ingedien vir die graad D. Ing in die Fakulteit Ingeniieurswes. Universiteit Van Pretoria, April 1984.

[29] MCGregor, P., Double diffused MOSFET's for C.A.T.V. Amplifiers, M.A. Sc. Thesis, Toronto, 1982.

[30] TAYLOR, G. W., «Velocity-Saturated Characteristics of Short Channel MOSFET's », The Bell System Techn. J. 63 (1984).

[31] Rossel, P., Propriétés statiques et dynamiques du transistor MOS, Thèse, UPS Toulouse 1973.

[32] Guegan, G., Contribution à l'étude des propriétés dynamiques du transistor Métal-Oxyde-Semiconducteur à canal vertical (V.MOS), Thèse Doc.Ing., UPS Toulouse, 1979. 
[33] Ueda, D., Takagi, H., Kano, G., A New Vertical Power MOSFET Structure With Extremely Reduced On-Resistance, IEEE Trans. ED ED.32, $\mathrm{N}^{\circ} 1$ (1985) 2-6.

[34] Hu, C., Optimum Design of Power MOSFET's, IEEE Trans. ED ED.31, $\mathrm{N}^{\circ} 12$ (1985) 16931700.

[35] TARDIvo, G., Le Transistor D.MOS Vertical pour l'amplification de Puissance en Haute Fréquence, Thèse de $3^{\mathrm{e}}$ cycle, UPS Toulouse, 14 janv. 1987.

[36] Gamboa, M., Tranduc, H., Phan Pham, T., Rossel, P., «L.F. Dependence of the OutputAdmittance in Short Channel $\mathbf{N}$ or $\mathbf{P}$ Power
MOS Transistors », IEEE Trans. ED 29, $\mathrm{N}^{\circ} 5$ (1982) 838-841.

[37] Rossel, P., Martinot, H., Zamorano, M., Propriétés statiques des transistors MOS de puissance à canal vertical: Cas du régime de non-pincement, Revue Phys. Appl., Tome 13, N ${ }^{\circ} 1$ (1978) 1-6.

[38] Gamboa, M., Sarrabayrouse, G., Tranduc, H., Rossel, P., «Influence de la température sur la vitesse limite des porteurs dans un transistor MOS à canal court », Rev. Phys. Appl., 15, N 5 (1980) 973-975. 